Mair's Geographischen Verlag, das Straßenkarten von Deutschland mit Hilfe der von der Firma Dornier $\mathrm{GmbH}$ entwickelten Präsentationssoftware Geogrid ${ }^{\circledR}$ visualisierte. Zielsetzung bei der Entwicklung der MilGeo-PCMAP war es, ein standardisiertes Produkt für die militärische Nutzung zur Verfügung zu stellen, das sowohl auf einem üblich ausgestatteten PC lauffähig sein sollte als auch in hochgerüsteten militärischen Systemen als Kartendarstellungswerkzeug implementiert werden konnte. Dabei sollten die im AMilGeo und von den Verbündeten produzierten militärischen Geoinformationen in Form von Raster-, Bild-, Vektor- und Höhendaten sowie georeferenzierten Ortsnamensverzeichnissen visualisiert und korreliert werden. Aufbauend auf dem Nukleus Geogrid ${ }^{\circledR}$ entwickelte die Firma Dornier $\mathrm{GmbH}$ im Auftrag der Bundeswehr eine Fülle zusätzlicher Funktionen, die der militärische Nutzer für seine dienstlichen Aufgaben, wie z. B. die Geländebeurteilung für die Marschplanung oder Übungsvorbereitung, benötigt.

Bedingt durch Geogrid ${ }^{\circledR}$ enthält die seit 1997 entwickelte Top50 im Wesentlichen die gleiche Grundfunktionalität und Bedienungsoberfläche wie die der MilGeo-PCMAP. Die in der Version 3 der Top50 verwendete neve Präsentationssoftware Geogrid ${ }^{\circledR}$-Viewer 1.0 beinhaltet neben der Grundfunktionalität die vom AMilGeo konzipierten und in der MilGeo-PCMAP implementierten Höhendaten- und Datenbank- sowie die erweiterten Grafikund GPS-Funktionen. Diese über die Grundfunktionalität hinausgehenden Funktionen sind lizenzrechtlich Eigentum der Bundeswehr. Ebenso war das AMilGeo an der Konzeptionierung der für die Version 4 der Top50 ge- planten Dynamischen Perspektivischen Darstellung beteiligt, die zurzeit in der MilGeo-PCMAP, Version 4.1, integriert wird.

Das AMilGeo hat stets der Entwicklung der Top50 aufgeschlossen gegenüber gestanden und diese des Ófteren auch tatkräftig mit einem Vertreter in der für die Entwicklung der Top50 zuständigen Ad-hoc-Gruppe "CD-ROM" der AdV beratend unterstützt. Man darf bei aller Bescheidenheit betonen, dass die MilGeo-PCMAP die Funktionalität der Top50 wesentlich mit beeinflusst hat lund nicht umgekehrt, wie manchmal behauptet wirdl. Gleichwohl partizipieren beide Produkte gegenseitig von Optimierungen vorhandener Funktionen auf Grund von Forderungen und Wün- schen der zivilen und militärischen

Nutzer.

Bei der sehr guten zivil-militärischen Zusammenarbeit der deutschen GeoDienststellen ist es sehr verwunderlich, dass diese K. Tönnessen bekannten Fakten urheberrechtlicher Relevanz in seinem Beitrag nicht genannt werden.

\section{Literatur}

Brech, W.; Scharf, K.-H.; Schlarb, R.: Die MilGeo-PCMAP - Möglichkeiten einer elektronischen Kartendarstellung. In: Nachrichten aus dem Karten- und Vermessungswesen, Reihe I, Heft 117, S. 9-12; Hrsg.: Bundesamt für Kartographie und Geodäsie, Franfurt/Main, 1997.

Anschrift des Verfassers: Dipl.-Ing. Klaus-H. Scharf, Amt für Militärisches Geowesen, Frauenberger Straße 250, D-53879 Euskirchen, Telefon: +49 2251953. 23 23, Fax: +49 2251 953- 23 11, E-Mail:

KlausScharf@bundeswehr.org

Klaus-H. Scharf, Euskirchen

\title{
Kommentar zur Stellungnahme von K.-H. Scharf
}

Die in der Sache zutreffenden Ausführungen von Klaus-H. Scharf stellen auf die enormen und zu keiner Zeit in Frage gestellten Vorleistungen sowie die richtungsweisenden Impulse für die Entwicklung der Basissoftware im Amt für Militärisches Geowesen ab. Ziel meines Beitrags in Heft 5/2002 war. nicht, die Hintergründe der Entwicklung des Geogrid ${ }^{\circledR}$-Viewers aufzuzeigen, sondern die Breite der Anwendungen eines auf dem freien Markt in bundesweiter Einheitlichkeit als Serie Top50 platzierten Produktes. Formal betrachtet war für die Produktionen der einzelnen Landesvermessungsstellen die Basissoftware Geogrid ${ }^{\circledR}$ in einer ausschließlich auf die zivile Nutzung nach fachlichen und verkaufsstrategischen Gesichtspunkten ausge- wählten Fassung jeweils Gegenstand der Produktions- und Lizenzvereinbarungen mit dem Hause Dornier.

Gleichwohl war es nicht Absicht des Verfassers, die im Vorfeld für die MilGeo-PCMap konzipierten und in wesentlichen Teilen beauftragten Entwicklungen zum Geogrid ${ }^{\circledR}$-Viewer zu verschleiern. Eine aus vermeintlichen Sicherheitsinteressen gebotene Zurückhaltung hinsichtlich Verlautbarungen zu militärischen Entwicklungen wird offenbar heute anders beurteilt. Demzufolge kann an dieser Stelle die überaus gute zivil-militärische Zusammenarbeit einmal mehr bestätigt und die veröffentlichte Stellungnahme somit als begrüßenswerte fachliche Ergänzung des Beitrags verstanden werden.

Karl Tönnessen, Bonn 